\title{
Filigrane
}

Écoutes psychanalytiques

\section{L'entrelacs des tracés de la violence dans les psychés et dans la culture}

\section{Ellen Corin}

Volume 25, numéro 1, printemps 2016

Actes du colloque Le sujet sacrifié (Ghyslain Lévy)

URI : https://id.erudit.org/iderudit/1037373ar

DOI : https://doi.org/10.7202/1037373ar

Aller au sommaire du numéro

Éditeur(s)

Revue Santé mentale au Québec

ISSN

1192-1412 (imprimé)

1911-4656 (numérique)

Découvrir la revue

Citer cet article

Corin, E. (2016). L'entrelacs des tracés de la violence dans les psychés et dans la culture. Filigrane, 25(1), 53-66. https://doi.org/10.7202/1037373ar 


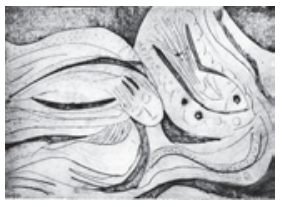

\section{L'entrelacs des tracés de la violence dans les psychés et dans la culture}

\section{Ellen Corin}

$\Lambda$ border la question de la violence nous oblige à nous situer au plus près de la face sombre de notre temps, au plus près aussi de l'obscurité qui nous habite comme humains. Si l'une et l'autre scènes, la collective et la psychique, ne sont en aucun cas superposables l'une à l'autre, il existe entre elles des résonances troublantes qui nous sollicitent comme analyste. Comment se laisser affecter par l'une et l'autre sans y perdre notre esprit, notre âme et nos mots? Comment penser les passages entre les deux scènes? Comment aussi se mettre à l'écoute de la clinique pour explorer notre réaction à ce qui se joue sur la scène de la culture, pour nous interroger sur la manière dont nous en sommes partie prenante, comme analystes et comme humains? Et quels chemins emprunter pour tenter d'affronter et d'élaborer ce qui fait trace en nous de la violence du monde?

C'est en écho à la violence et aux atrocités de la Première Guerre mondiale que Freud écrit ses Actuelles sur la guerre et sur la mort (1915), un texte dans lequel il s'interroge sur l'inévitabilité de la guerre dans les sociétés. Cinq ans plus tard, dans Au-delà du principe de plaisir (1920), Freud franchit un pas de plus et cherche à cerner la place de la violence dans la psyché, à travers l'hypothèse d'une pulsion de mort constitutive de la vie psychique au même titre que la pulsion sexuelle. C'est donc dans une même mouvance que Freud s'interroge sur la violence qui prévaut dans la culture et dans les psychés.

Loin de la réduire à l'agressivité, Freud parle de la pulsion de mort comme d'une tendance à une déliaison radicale qui s'attaque avant tout à la vie psychique elle-même, à la possibilité de faire des liens et de former des représentations, une pulsion qui vise un retour à l'inertie et à l'inorganique. Une pulsion de mort active en chacun de nous, mais on peut faire l'hypothèse que la violence vécue sur la scène externe peut rejoindre et exacerber cette violence interne qui nous constitue. Une pulsion de mort œuvrant en 
silence au sein de la psyché et que le masochisme érogène primaire va chercher à lier au début de la vie, nous dit Freud (1924), un masochisme qui se pose alors comme gardien de la vie face à la destructivité pure de la pulsion de mort. Nous savons cependant à quel point l'investissement masochique de la souffrance attachée à la violence pose un terrible obstacle à la thérapie. Freud remarque aussi que la pulsion de mort peut se lier au mouvement qui porte la libido vers l'objet, profiter de son énergie pour se diriger vers le monde. Dans la clinique aussi, nous sommes témoins et parfois victimes de ce retournement de la pulsion de mort vers l'extérieur, une violence associée alors à une jouissance dont témoigne un certain éclat du regard.

La pulsion de mort est essentiellement muette, nous dit Freud, et c'est à travers son intrication avec la pulsion sexuelle qu'elle nous devient visible. C'est donc à travers le fil de cette intrication que nous pouvons en percevoir les effets, même lorsqu'une absence d'associations, de mots ou d'images nous donne l'impression d'être en présence d'un territoire dévasté, d'être confrontés à une pulsion de mort "à l'état pur». Une des manières dont cette intrication-passeur fait signe sur la scène clinique passe par des représentationschose, des représentations-chair pourrait-on dire, au plus près du corps.

Du côté de la pulsion de mort, je pense à un film récent de Bela Torr, Le cheval de Turin. C'est un film lent, muet, en noir et blanc, de 146 minutes. Jour après jour, durant six jours, se reproduit inchangé le rythme des quelques gestes lents qui scandent le quotidien d'une fille et d'un père dans une chaumière misérable au milieu d'un paysage désertique balayé par un vent violent. Un à un, les signes associés à la vie s'effacent: le cheval refuse la routine habituelle, harnachement et marche; l'eau du puits se tarit; la tentative de départ du père et de la fille tirant une charrette se révèle vaine lorsqu'on voit leur silhouette revenir se détachant sur la crête d'un paysage désolé; et à la fin, c'est la lumière elle-même qui s'efface lorsque la lampe à huile et le feu s'éteignent et que le père et sa fille demeurent face à face dans l'obscurité. On est proche ici du retour à l'inertie et à l'inorganique que Freud associe à la pulsion de mort.

Et pourtant, il y a ce vent, un vent insensé, qui pourrait rendre fou. Et aussi le fait que tout le film est habité par une sensorialité qui nous rejoint au plus près du corps. Derrière la violence et la mort, et même dans cette situation de désolation extrême, le feu du sexuel. Et en toile de fond, la couverture du dernier livre de Ghyslain Lévy (2014), sur laquelle c'est l'ombre projetée de la bougie éteinte qui porte la flamme alors que la bougie ellemême semble morte. 


\section{Sur la scène clinique}

Sur la scène de la clinique, il y a des moments où non seulement les mots font défaut mais où le corps semble lui aussi basculer dans l'inertie qui a envahi la vie psychique, côtoyant un risque de mort bien réel. L'impression alors d'un surgissement de la pulsion de mort dans sa dimension de déliaison radicale, une présence nue dans laquelle il faut pouvoir se tenir sans le secours d'interprétations trop vite formulées et qui ne pourraient que tomber en porte à faux par rapport à ce qui a lieu. Se tenir là sans en mourir et, prenant de plein fouet l'effroi partagé, rendre possible la réouverture d'un chemin vers la figuration et le langage.

Si on revient à la question des traces de la violence dans la psyché, il faut sans doute repartir du fait que la première forme de violence est celle du pulsionnel lui-même, un pulsionnel qui tend à la décharge et que libère paradoxalement la règle de la libre association dans la cure psychanalytique. Celle-ci défait la cohérence du récit et permet le surgissement d'autres associations, de vécus anciens, d'images sensorielles qui plongent leurs racines dans un passé lointain. Ainsi, derrière la langue articulée du récit se fait jour une langue-affect plus brute, qui ne se réfère pas à un objet particulier et ne s'adresse à personne, la langue de l'infantile qui fait surface à la faveur du transfert, un infantile qui vient déranger le cours des séances.

C'est par ce sensoriel que peut se faire jour quelque chose qui résiste aux représentations et aux mots, un sensoriel qui déroute le champ des représentations initiales et trace la voie au surgissement d'autres images, d'autres mots davantage en prise avec la réalité psychique. De telles images sensorielles sont susceptibles d'animer les tracés figés qu’a imprimés la violence dans la psyché, de les détacher de l'effet de sidération accompli par la violence première.

Il importe aussi d'avoir toujours à l'esprit que, quelle que soit sa sauvagerie, la violence ne se transpose jamais telle quelle dans la psyché, qu'elle y est reprise, infléchie, rejouée à travers le jeu interne des pulsions de vie et de mort. La seule réalité à laquelle nous ayons réellement affaire, la seule qui nous concerne, est la réalité psychique dans laquelle ce qui pouvait paraître monochrome ou déshabité lors des premiers contacts peut se mettre à prendre des couleurs changeantes, souvent déroutantes.

Le travail d'analyse se déplace ainsi progressivement le long de la surface que dessine l'entrelacs entre réalité matérielle et réalité psychique. Catherine Chabert (2015) nous rappelle qu'autant il est important en début d'analyse d'être attentif à la «vérité» de l'histoire matérielle, autant il importe de 
progressivement prendre en compte le fonctionnement psychique de chaque individu et la manière dont il est susceptible de traiter les événements de la vie; et autant il importe de permettre le dévoilement de cette autre scène où s'actualisent les fantasmes.

Cette réalité psychique apparaît le plus souvent inaccessible au début de la thérapie, alors que la violence se manifeste dans des agirs ou que l'on a l'impression de se trouver face à un désert de représentations, d'images et de mots. Ces agirs répétitifs peuvent aussi revêtir l'allure d'un destin lorsque les mêmes impasses, les mêmes épreuves se répètent non seulement dans la vie de la personne mais aussi à travers les générations. Et lorsque des images surgissent, elles semblent souvent immobiles, répétitives, porteuses d'un effet de sidération plus que d'ouverture.

Parfois, pour évoquer ce qui la hante, la personne emprunte à la réalité extérieure des images ou des événements qui servent de tenant lieu pour ce qui est hors représentation ou hors image, ou pour évoquer de biais la menace d'un débordement trop dangereux pour pouvoir être reconnu en première personne. Je pense ici à un patient qui passe parfois de longs moments des séances à évoquer la corruption ou la perversité des figures d'autorité, dans un récit à la fois réprobateur et fasciné qui ouvre de manière oblique sur la manière dont la violence qui a marqué les premières années de sa vie impliquait un nouage entre la violence et le sexuel. Je l'écoute et en contrepoint de son récit, je l'entends me parler d'une réalité intime, qui le tourmente et l'obsède. Ce qui prend alors corps est moins la violence de l'événement passé que son empreinte sur la psyché. Laisser alors se déployer ces images, les écouter de deux oreilles à la fois, l'une tournée vers cette réalité externe qui nous est adressée, et l'autre vers la réalité psychique dont elle est l'écho.

Il arrive aussi que d'emblée, la personne nous offre un premier récit de violences passées ou présumées, même si parfois, ces événements passés sont affectés d'un caractère d'incertitude, une incertitude qu'elle tente de réduire en demandant confirmation de souvenirs en fragments à des membres de son entourage. Dans ces premiers récits, les rôles de victime et de bourreau, les caractéristiques des personnages, ce qui a trait à la souffrance et à la jouissance paraissent souvent relativement bien démarqués. Au cours de l'analyse, cette première mise en scène se révèle un écran qui masque autant qu'il ne révèle, un écran sur lequel en vient à se projeter progressivement un monde fantasmatique beaucoup plus complexe et ambigu, un chatoiement dans lequel le pourpre et le noir, le désir et la colère sont étroitement 
imbriqués et où les jeux de l'agression et de la séduction, de la pulsion de mort et d'Éros ne se laissent plus réduire à des logiques binaires.

Ce sont d'autres modalités d'images, au plus proche de la sensorialité, qui servent souvent de passeur sur la scène clinique; des images qui présentent à la perception quelque chose qui était demeuré informulé, une frange affective de la réalité psychique qui était restée hors langage. Peu à peu, la surface que constitue le désert apparent des représentations se voit agitée de remous, alors que des images ou des bribes de souvenir se font jour.

Au cours de certaines séances avec un patient, j'ai eu l'impression que le langage tombait en pièces: ce n'était pas seulement ce que nous avions essayé de construire ou de lier au cours de séances précédentes qui se voyait défait, mais les mots eux-mêmes, les miens, qui semblaient devenir des «objets volants non identifiés ", des signifiants flottants déconnectés de tout pouvoir de signification. Une violence faite à la langue, venant rejouer en séance, au niveau des mots, la violence première faite à la psyché alors qu'une détresse originaire n'avait pas trouvé d'écoute ou d'abri pour calmer l'angoisse.

Ou encore, l'impression d'angoisse à la fois insistante et diffuse qui s'est imposée au cours d'une séance, alors que la patiente notait un changement dans la couleur des murs, le fait que le cadre rassurant que formait l'ombre d'une fenêtre évoquait maintenant les barreaux d'une prison, et qu'elle me voyait en pensée lui tournant le dos: une angoisse surgissant «en présence», par le biais d'indices perceptifs donnant "forme informe» à une détresse originaire ouvrant sur un sentiment d'abandon et sur un effroi face à la mort. Un affect surgissant dans l'actuel, au niveau d'un ressenti partagé.

D'autres souvenirs de telles traces sensorielles me reviennent. Comme la forte angoisse, mentionnée par plusieurs personnes, à l'évocation de l'eau disparaissant dans le fond d'une baignoire, comme aspirée par le vide, comme si était donné là à voir et à ressentir un risque d'engouffrement et de disparition; une représentation primitive proche du pictogramme dont parle Piera Aulagnier (1975). Ou encore, sur un plan apparemment moins archaïque, l'image d'un frigo qui s'est imposée à une descendante de génocide et qu'elle fait jouer tout au long de sa thérapie pour en explorer les diverses facettes et s'en servir comme d'un élément traceur qui lui permet d'explorer la gamme des affects associés au fait d'avoir été confrontée directement à un risque personnel de mort dans une enfance en guerre, à l'effroi non parlé du génocide passé, à la solitude de l'exil.

Des moments importants, difficiles, qui captent dans l'actuel de la séance la trace sensorielle de l'effet de la violence sur la réalité psychique. 
C'est comme s'il fallait passer par cette réactualisation des traces pour que quelque chose vienne habiter la langue, pour que la langue devienne dès lors porteuse d'effets de sens et que la réalité psychique se fasse plus mobile, ouverte au changement, qu'un autre récit, habité, puisse venir aux mots.

Il ne s'agit pas alors, depuis sa position d'analyste, d'interpréter la trace ou de la traduire en mots, mais de favoriser ses déplacements et son mouvement vers la figuration. De rendre la trace à la texture de la réalité psychique qu'elle exprime et d'en permettre une remise en jeu dynamique. Ainsi, c'est en prenant appui sur l'énergie du transfert que progresse la recherche d'une figuration de ce qui œuvre dans la psyché.

Il existe donc une tension entre un mode de figuration qui demeure pris dans l'événement traumatique et autre mode de figuration, animé par la vie fantasmatique. Alors que le premier de ces modes demande à l'analyste de demeurer là, en présence lui aussi, de prendre acte des tracés de la violence qui ont creusé leur empreinte dans la psyché, d'agir comme témoin, le second laisse plus de place à l'interprétation et au soutien offert à l'élaboration d'un autre récit qui à la fois fasse sens et qui ouvre. Dans le premier cas, l'analyste peut se trouver captif d'un effet de sidération, demeurer pris dans un «arrêt sur image», ou être tenté de se poser en sauveur, sans pour autant échapper à l'impuissance de cette position. Dans le premier cas aussi, la tentation est grande de donner nous-mêmes sens aux images ou aux traces, y projetant nos propres fantasmes, sans réaliser que ces traces s'inscrivent dans des réalités psychiques qui nous échappent.

Si l'on revient à la question de l'intrication des pulsions, on peut dire que certaines images semblent demeurer prises dans le mortifère de la pulsion de mort alors que d'autres semblent habitées par un mouvement animé par la pulsion de vie.

\section{Sur la scène collective}

Ce sont des dynamiques similaires qui me paraissent jouer sur la scène collective. Une patiente dit: "Le monde extérieur me rentre dedans», et la perte de repères fiables au dehors entre en phase avec le sentiment que sa réalité intérieure bascule, que la bulle qu'elle avait construite autour d'elle se voit effractée et risque de tomber en pièces.

Dans le monde actuel, on a l'impression d'être envahis par un surplus d'images et de mots, particulièrement d'images qui présentent à l'état cru la barbarie qui menace toujours de mettre en péril le travail de la culture. Des 
images qui nous convoquent à une position de témoin mais avec le risque que nous nous retrouvions sidérés, captifs de la violence des images, ou encore que nous cédions à la tentation de nous poser en sauveur sans nous intéresser d'abord à l'arrière-plan de ce dont nous percevons l'effet.

Certaines images nous laissent dans une attraction pétrifiée. Je pense aux décapitations par Daech, des exécutions qui visent à produire un effroi qui se trouve décuplé lorsque les victimes sont présentées au public, désanonymisées, une identification potentielle à la victime s'ajoutant alors à la barbarie visible de l'acte. Une réaction d'horreur qui n'est pas exempte de fascination comme en témoigne la circulation des images sur le web. D'autres fois, nous sommes conscients qu'une violence aussi grande est liée aux "effets collatéraux» d'une guerre se voulant "plus propre» comme la guerre des drones. Mais cette fois, l'absence d'images donnant accès à cette violence seconde produit en nous un effacement de l'horreur. Plus près de nous, on pense à la manière dont le niqab, vêtement hautement improbable dans notre société, s'est introduit dans les débats électoraux et en a largement faussé les enjeux, reléguant à l'arrière-scène des thèmes aussi importants que les inégalités sociales ou le détournement de la recherche. Dans ce cas, mais aussi dans les autres, l'image qui nous est proposée semble agir comme un écran de captation d'un imaginaire collectif et personnel fait de craintes latentes et de sourdes colères.

Ainsi, sur ce premier versant, les images jouent doublement un rôle d'écran: comme ce qui masque et qui fige, et comme ce sur quoi on projette une réalité psychique qui nous est insue et qui parle essentiellement de nous.

Il y a aussi bien sûr l'image de l'enfant syrien mort sur la plage, comme il y avait eu celle de l'enfant guetté par un vautour captée par le fils de Jimmy Carter. Des images dont on voudrait qu'elles contribuent à remettre en mouvement notre rapport intime à l'autre en tant qu'il est objet de violence mais dont le pouvoir se trouve rapidement érodé, comme si les images singulières se trouvaient alors versées au compte du surplus d'images qui nous envahit et contre lesquelles nous nous protégeons tout comme nous nous protégeons de la violence qui nous habite.

Face au pouvoir des images, se dresse la force du témoignage lorsqu'il repose sur une proximité avec le monde de l'autre et que l'image s'anime, se greffe sur un récit. Mais le témoignage n'est jamais simple et lorsqu'il s'agit de témoigner du mal, le risque est toujours grand, comme sur la scène clinique, de se présenter en héros ou de se laisser prendre par les sirènes silencieuses et terribles de la mort en direct. 
Deux films me viennent ici à l'esprit. Le premier est Le sel de la terre, de Wim Wenders, qui nous introduit à l'univers du grand photographe brésilien Sebastiao Salgado. Si l'on excepte sa dernière partie, salvatrice, ce film apparaît tendu entre deux pôles: au départ, un voyage vers des terres et des gens à la fois lointains et proches, lointains par «l'étrangèreté» de leur apparence et de leur mode de vie, de leurs costumes; proches par l'éclat d'humanité partagée que reflètent les regards. De l'autre, l'atrocité dans laquelle nous plongeons avec l'auteur alors que, s'intéressant à l'exil, il se heurte à l'effet ravageur des grandes famines et des guerres en Afrique de l'Est, puis à celui des meurtres de masse au Rwanda et plus tard, au Congo, puis aussi à ces scènes d'exilés chassés de chez eux par les affrontements dans les Balkans. Des scènes dont Salgado capte l'horreur et dont il se/nous constitue en témoin.

Ce qui me frappe est que la traversée de l'humain que poursuit le photographe et dont il capte magnifiquement les reflets par ses photos, le mène progressivement à se confronter à la violence inhérente à l'homme, une violence qui semble projeter hors de l'humain tant le bourreau que la victime, et qui atteint un tel degré d'insoutenable qu'elle amène Salgado à ce constat terrible: «l'homme ne mérite pas d'exister». Même si l'on assiste dans la dernière partie du film à la lente renaissance de la vie en lui, on ressort du film avec un effroi lié à l'impression d'avoir, par le biais des images de Salgado, plongé au cour de la barbarie. Un effroi qui nous atteint à l'être.

On peut imaginer que ce constat que fait Salgado de la barbarie au cœur de l'humain le concerne aussi par un effet de miroir et interroge sa participation indirecte à l'œuvre de violence dont il témoigne. Comment porter témoignage de l'inhumain sans participer à sa diffusion et à sa mise en spectacle? Et plus profondément encore, comment témoigner de la violence sans se trouver capté par son aura? Comment regarder ces images, entendre ces récits au plus proche de l'humain, comment en saisir la texture faite de souffrance, de résistance et d'espoir?

Ce dilemme se trouve au cœur d'un autre film récent, L'épreuve, dans lequel Juliette Binoche joue le rôle d'une journaliste de guerre qui couvre l'Afghanistan. Le film s'ouvre et se referme sur deux scènes similaires, la préparation rituelle d'un corps de femme, de fillette à la fin du film, en vue de sa transformation en bombe vivante. En suivant le périple de la journaliste, on a l'impression que la nécessité de témoigner s'infléchit progressivement vers la fascination, une fascination qui la pousse à repartir vers le danger sans tenir compte de la détresse vécue par ses proches. Dans une entrevue, 
Juliette Binoche commente: "Je pense qu'un photographe de guerre va jusqu'à la limite de son possible, jusqu'à ce que son intuition lui dise: "Arrête ici parce que tu risques ta vie." Mais il y en a qui perdent la vie parce qu'il y a aussi un instinct de mort qui frôle certainement ce désir d'être toujours dans un monde dangereux, de se trouver dans les zones les plus dangereuses de la terre.» (Imbert, 2015)

Le mort et le vif, le sexuel et la mort entrelacés l'un à l'autre dans une intrication qui soutient d'un côté la possibilité de s'identifier à l'autre victime, de s'émouvoir et de protester; et de l'autre, qui nous tire du côté d'une sidération qui nous empêche de penser et d'agir. On peut rappeler ici que Nathalie Zaltzman place l'effroi à la source de la pulsion de mort. Il importe alors de réanimer des traces sensorielles plurielles, colorées, multi-centrées, tissées d'images, d'impressions et de fragments de récits, pour redonner sa place à un travail de culture susceptible de contrer la barbarie dans le social et en nous.

Tout comme sur la scène clinique, la nouvelle mise en récit que permet l'analyse laisse un reste que peuvent réactiver des circonstances de la réalité extérieure, le traitement collectif de la violence laisse un reste qui risque toujours de refaire surface sur le mode d'agir susceptible de faire irruption dans le tissu social. C'est peut-être sous cet angle qu'il faut considérer les tueries collectives qui surviennent à répétition chez nos voisins du Sud, formes contemporaines de la résurgence de violences non traitées du passé; ou encore, la violence de certains affrontements dans l'Afrique contemporaine, scories laissées par la barbarie de l'œuvre civilisatrice.

\section{Sur la scène de l'art}

Ce résidu non traité des violences collectives trouve un lieu d'expression privilégié dans les œuvres de certains artistes ou écrivains contemporains. Se mettre à l'écoute de ces œuvres, et surtout des traces sensorielles qu'elles mobilisent, nous permet un accès oblique, non frontal, à la violence et ouvre la voie à un travail de sensibilité et de pensée; à un travail de culture.

Dans ses réflexions sur le Mal, Nathalie Zaltzman (2007) parle du gain de lucidité à travers lequel le moi civilise le surmoi, le rendant moins inflexible. Elle ajoute: «Le mal saisi par une œuvre de création apporte un gain d'intelligence, un élargissement du moi, et même une prime esthétique qui a "la beauté du diable". ( (Zaltzman, 2007, p. 94)

Une œuvre de création et de dévoilement qui peut passer par les mots de l'écrivain, par les traits et les couleurs du peintre, par l'immobilité ou le mouvement des corps dans la danse. 
Les livres de Tony Morrison illustrent ce mouvement sur le plan de l'écriture. L'auteure est animée par le souci de rendre sensible, par l'imagination, tout un pan de l'histoire américaine qui a trait à l'esclavage et qui est largement occulté par l'Histoire officielle. Pour Toni Morrison, seul l'art peut se lever pour témoigner de ce dont l'histoire ne peut rendre compte. Seul l'art peut «enlever la cataracte» qui obscurcit le regard. Seule l'imagination peut pénétrer des domaines effacés de l'histoire.

Dans ses romans, elle fait non seulement revivre la réalité de l'esclavage, elle nous rend sensible la face subjective, douloureuse de la violence, l'atteinte à l'humain qu'elle produit.

Dans plusieurs de ses romans, le récit chemine en va-et-vient qui courtcircuite le temps chronologique, alors qu'effets d'avant-coup et d'aprèscoup s'entre-tissent en des séries complexes qui constituent une temporalité signifiante en regard de la réalité psychique. Il est aussi remarquable que dans ses romans plus récents, Home (2012) et Délivrances (2015), on voie la violence affleurer dans l'ère contemporaine, résurgence de ces restes hors langage; leur portée semble ici dépasser les affrontements de couleur même si la couleur en apparaît toujours comme la trame sous-jacente. En même temps, son écriture se simplifie, se fait plus épurée, plus proche de ces traces sensorielles dont j'ai parlé. Et dans Délivrances, la stigmatisation associée culturellement à la couleur de peau se noue avec la violence terrible du rejet par une mère qui ne supporte pas le foncé de la couleur de sa fille, dès sa naissance: «Elle m'a fait peur, tellement elle était noire. Noire comme la nuit, noire comme le Soudan [...]. Vous pourriez croire qu'elle nous renvoie en arrière, mais à quoi ?» (Morrison, 2015, p. 13) Et lorsqu'un sentiment de détresse profonde s'empare de la fille et la met en mouvement, sa régression vers un état infantile se marque dans son corps par la disparition de ses poils pubiens, puis de ses marques de boucles d'oreille, par son retour à une maigreur d'enfance, par l'effacement de ses seins. Une régression qui trace son trajet à même le corps; une figuration de la dramatique humaine au plus proche du corps.

C'est par le biais d'une déconstruction de l'écriture même que d'autres écrivains cherchent à rendre sensible l'effroi associé à l'ère contemporaine, qu'il s'agisse par exemple de la contemporanéité de la Shoah avec Paul Celan ou de celle d'Hiroshima avec de jeunes écrivains japonais. Mais de cela, Ghyslain Lévy (2010) a magnifiquement parlé et je veux juste l'évoquer ici.

Dans un autre registre, dessins et peintures permettent aussi le surgissement des traces d'un infigurable situé au-delà de ce que le récit peut évoquer 
d'une violence collective. Toutefois, ici comme dans le cas des écrivains, et comme le remarque Yves Bonnefoy (2006) dans ses commentaires aux œuvres noires de Goya, ni les faits de la société ambiante ni les événements de la biographie ne suffisent à rendre compte de l'œuvre. Ce qui s'exprime à l'occasion de ces éléments de réalité met en jeu un niveau plus profond de la relation à soi et à l'humain. Et si l'histoire joue aussi son rôle dans cette figuration, note Yves Bonnefoy, c'est que les grands événements qui affectent l'histoire sont eux-mêmes d'une nature profonde et qu'ils concernent aussi une crypte de l'esprit « où rien ne motive sinon des pulsions, des aspirations, des pressentiments, que les philosophes ni même les poètes ne parviennent à formuler» (p. 11). Il parle d'une "pensée figurale» qui vise à «aller à la vérité, et l'affronter, dans son lieu qui est au-delà des formulations et reconstructions qui fleurissent dans le discours» (p. 12).

Les dessins et peintures de Vladimir Velickovic (Catonné, 2014), un peintre d'origine yougoslave, nous ouvrent un accès privilégié à l'empreinte que la violence collective laisse dans la psyché. Hantée par les atrocités commises par les nazis dans un Belgrade sous les bombes, toute son œuvre exprime la douleur et la violence de cette mémoire. Ce qui m’intéresse particulièrement ici, est le double tracé que suivent ces œuvres.

D'un côté, d'immenses dessins à l'encre de Chine, rouge et noire, disent l'excès de l'acharnement sur les corps alors que s'entremêlent des rats énormes, des dogues/pit-bulls hurlants, des bandes de corbeaux, des corps pendus, écartelés, tombant, engouffrés, entrelacés dans un horrible magma dont chaque détail nous est rendu avec une précision quasi-chirurgicale qui nous rend sensible à l'effroi.

De l'autre côté, il y a ces tableaux eux aussi immenses, d'une sobriété extrême: ciels sombres avec au fond le rougeoiement d'incendies lointains et souvent des corbeaux immenses, occupant tout le devant la toile, ou encore des corbeaux dont les silhouettes se devinent dans la brume alors qu'ils s'abattent en masse vers le sol. Un espace qui, commente l'auteur, dit la disparition de la présence humaine et une nuit, un crépuscule qui rendent sensible une absence infinie. De ses peintures, Velickovic dit qu'elles font «voir comment vibrent les situations».

Ses dessins à l'encre de chine sont pour lui une nécessité quotidienne, «l'équivalent même de son existence» et sans eux, remarque-t-il, il se mettrait à assommer tout le monde; le trait qu'il trace lui fait ressentir «une excitation que l'on ne peut décrire avec les mots». Le cadre de ses peintures est tout autre. Ce sont des œuvres de longue haleine qu'il travaille et 
retravaille jour après jour, pendant des mois parfois, avec les mains, avec les paumes, avec un pinceau grossier.

Ainsi, le trait aigu des dessins cruels semble directement en prise avec les tracés de la violence dans le corps, une violence que le peintre aurait incorporée, faite sienne, avec toute leur cruauté, en ce lieu où la sauvagerie du sexuel fait alliance avec la mort. Les peintures me semblent traiter cette violence à travers le sublime; le caractère insoutenable de ce qui a eu lieu et son effet de dévastation s'expriment à travers la convocation de la beauté infinie et terrible du mal.

Ainsi existent, côte à côte, l'aigu de la blessure, son inscription dans le corps et le travail de son élaboration: le cri de la plume sur le papier, avec la sensualité que peut convoquer la violence; et la sensorialité d'une peinture longuement appliquée, à main nue. Nul salut d'un côté comme de l'autre, mais une urgence de porter témoignage, moins des événements de violence que de la blessure.

Georges Didi-Huberman (2007) parle d'une image ouverte pour désigner une économie très particulière de l'image, «un événement d'image où se déchire profondément, au contact d'un réel, l'organisation aspectuelle du semblable»; un moment où apparaît tout à coup un dissemblable essentiel, l'image même de la dislocation. Une image qu'il distingue de la plupart des images qui nous environnent et qui ne proposent «qu'écrans, bouche-trous, sutures par le semblant» (Didi-Huberman, 2007, p. 35).

Les images ouvertes nous interpellent et leur ouverture est indissociable de notre participation au travail de l'image: «Nous sommes devant les images comme devant d'étranges choses qui s'ouvrent et se ferment alternativement à nos sens... elles s'ouvrent à nous et se ferment sur nous dans la mesure où elles suscitent en nous quelque chose que l'on pourrait nommer expérience intérieure.» (Didi-Huberman, 2007, p. 25)

Je pense ici à certains dessins à travers lesquels, chez les Impatients, s'exprime la violence hors langage qui est la face interne, sombre de leurs problèmes de santé mentale. La violence du trauma; celle qui s'exprime dans le cri et dans le mutisme; celle que portent les regards d'autrui.

L'idée de l'intrication entre pulsions de vie et pulsions de mort nous ramène aux débuts de la vie psychique, à sa genèse et à son rapport à la mort. Ici, la violence concerne l'émergence de la vie sur fond d'inanimé, ce moment où une forme commence à se dégager de ce qui auparavant semblait informe. La Bible dit: "Yhwh Dieu fabrique un Adam poussière qui vient du sol, souffle la vie dans ses narines, l'Adam se met à vivre.» (Genèse, 2 :7) 
De cette lente émergence d'une forme, de ce moment de genèse primordiale, la danse japonaise du Butoh nous offre une figuration visible. Se développant au croisement entre un rejet de l'esthétique traditionnelle japonaise et un intérêt pour les avant-gardes européennes des années cinquante, le Butoh retrouve des racines profondément japonaises tout en ayant une portée universelle. Tamotsu Watanabe (2003) commente que le détour par l'Orient qu'implique le Butoh nous amène dans les profondeurs, par le biais d'une pensée qui cherche à dépasser le visible et à voir ce qu'on ne peut pas voir. Se développant dans un contexte marqué par le pessimisme qui règne dans le Japon des années soixante, il est animé par une "quête des origines du corps obscur». Odette Aslan (2004) écrit: «Se revendiquant marginal et paria, le danseur prend sur lui le mal de la société en assumant les indignités, en descendant vers l'abîme du Mal pour y trouver, à la manière de l'écrivain maudit Jean Genêt, la source de la régénération et d'une forme de sainteté.» (Aslan, 2004, p. 20)

Nathalie Zaltzman (1998), pour revenir à cette auteure en conclusion, voyait le processus de guérison accompli par la psychanalyse comme une remise en mouvement d'un travail de culture. Un travail qui passe nécessairement par une remise en jeu de notre fond pulsionnel et la tentative de l'élaborer. Un travail de culture qu'accomplissent de leur côté, à leur manière, écrivains et artistes contemporains. Ainsi l'art, allié de la psychanalyse sur la scène collective.

Nathalie Zaltzman (2007) conclut son livre L'esprit du mal en revenant sur le livre de Golding Sa Majesté des Mouches, alors que l'esprit du mal chuchote à l'oreille de l'enfant: "Tu savais bien, voyons, que je faisais partie de toi. Intimement partie de toi, intimement. Je suis ce qui fait que rien ne va, tu le sais.» (p. 26) Elle ajoute:

Le travail de culture est ce savoir intime. Il ne suffit pas que le sachent les individus, un à un. Il faut aussi que l'humanité, celle qui se purifie de ses propres crimes en se sacralisant, réussisse à «connaître» l'intimité en elle de la dimension du mal. (p. 110)

Ellen Corin

ellen.corin@gmail.com 


\section{Références}

Aslan, O. (2004). Introduction. Dans O. Aslan et B. Picon-Vallin (dir.), Butô(s) (p. 16-24). Paris: CNRS Éditions.

Aulagnier, P. (1975). La violence de l'interprétation. Paris: Presses universitaires de France. Bonnefoy, Y. (2006). Goya, les peintures noires. Mayenne: William Blake \& Co Édit.

Catonné, F. (2014). Vladimir Velickovic, le choix du noir. [Film cinématographique] Paris.

Chabert, C. (2015). La jeune fille et le psychanalyste. Paris: Dunod.

Didi-Huberman, G. (2007). L'image ouverte. Paris: Gallimard.

Freud, S. (1915). Actuelles sur la guerre et sur la mort. Euvres complètes tome XIII - 19141915 (p. 125-160). Paris: Presses universitaires de France, 2005.

Freud, S. (1920). Au-delà du principe de plaisir. Euvres complètes tome XV - 1916-1920 (p. 273-338). Paris: Presses universitaires de France, 2002.

Freud, S. (1924). Le problème économique du masochisme. Euvres complètes tome XVII - 1923-1925 (p. 9-23). Paris: Presses universitaires de France, 1992.

Imbert, T. (2015). «L'Épreuve»: 3 questions à Juliette Binoche. [Vidéo en ligne]. Repéré à http://www.allocine.fr/article/fichearticle_gen_carticle=18641529.html

Lévy, G. (2010). L'ivresse du pire. Paris: Campagne Première.

Lévy, G. (2014). Le don de l’ombre. Paris: Campagne Première

Morrison, T. (2012). Home. Paris: Christian Bourgois.

Morrison, T. (2015). Délivrances. Paris: Christian Bourgois.

Watanabe, T. (2003). L'orient dans le miroir. Dans U. Amagatsu et G. Delahaye (dir.), Sankai $J u k u$ (p. 7-9). Paris: Actes sud.

Zaltzman, N. (1998). De la guérison psychanalytique. Paris: Presses universitaires de France.

Zaltzman, N. (2007). L'esprit du mal. Paris: Éditions de l'Olivier, penser/rêver. 